\title{
Sleep science in anesthesiology
}

\author{
Tetsuya Kushikata ${ }^{1}$ \\ Received: 29 March 2019 / Accepted: 2 April 2019 / Published online: 6 April 2019 \\ (c) Japanese Society of Anesthesiologists 2019
}

Keywords Sleep $\cdot$ Anesthesia $\cdot$ Sleep disturbance $\cdot$ Mechanism of general anesthesia

The discipline of sleep science offers two merits to anesthesiology: a basic aspect and a clinical aspect. The basic aspect's merit is that sleep science provides knowledge that can be useful to elucidate the mechanism by which general anesthesia works, especially concerning the loss of consciousness. On the other hand, the clinical aspect's merit is that knowledge of sleep science may provide answers to solving postoperative sleep disturbance, which is widely recognized as one of the established factors that exacerbates problems associated with the postoperative course.

Anesthesia-induced amnesia is a distinct state from physiological sleep, especially considering that general anesthesia has the potential to be harmful. If we develop a method of anesthesia that results in a state of amnesia similar to physiological sleep, it may be beneficial to patients.

\section{Anesthesia and sleep: from the viewpoint of basic medicine}

There are a number of general anesthetics including gaseous, inhaled, and intravenous anesthetics. All of these anesthetics have distinct physical and chemical properties. Therefore, general anesthesia includes various agents that induce a reversible state of altered consciousness that satisfies the following conditions: loss of consciousness, analgesia, muscle relaxation, and the suppression of autonomic nervous reflexes.

The strategy for basic research on anesthesiology is divided into the macro-to-micro hierarchy. We have yet to discover a uniform theory that is able to explain all

Tetsuya Kushikata

tetsuyak@hirosaki-u.ac.jp

1 Department of Anesthesiology, Hirosaki University Graduate School of Medicine, Zaifu 5, Hirosaki, Aomori 036-8562, Japan anesthesia-induced phenomena across the entire hierarchy. At the micro level, many studies investigated how anesthetics affect the dynamics of the consciousness-related quantum [1], molecules, classical neurotransmitters, and modifiers. Recently, the trend has been to clarify how general anesthetics affect consciousness-related neuronal network systems $[2,3]$.

On the other hand, there are also a number of excellent studies that investigated the mechanism of general anesthesia by identifying common points between sleep and anesthesia. For example, a revolutionary study by Nelson et al. discovered that GABAergic anesthetics, dexmedetomidine as well as GABA agonist itself, all induced a similar neuronal activity pattern, known as c-fos expression, in the socalled endogenous sleep circuit regardless of whether the animal was anesthetized or sleeping [4].

Currently, we have two theories for the mechanism by which general anesthesia works. The first, the so-called bottom-up paradigm, explains that general anesthetics act on various nuclei in the brain responsible for sleep states and awakening from those states, resulting in a loss of consciousness. The top-down paradigm, on the other hand, argues that modulation of signal transmission of neural networks between the cerebral cortex and the thalamus causes loss of consciousness. The issue of which of these paradigms is superior is still under investigation [3, 5-7]. Both theories agree that the disconnection of information transfer in the central nervous system is one of the essential mechanisms of general anesthesia-induced loss of consciousness. In contrast, only Akeju and Brown [8] propose that general anesthesia is neurophysiologically distinct from sleep, because the changes in EEG patterns are anesthetic agent dependent and some agents produce different EEG patterns from sleep.

Anesthesia studies that utilize sleep science have revealed that the process of induction and emergence from anesthesia is not a simple mirror of the unitary mechanism. Previous studies have established that physiological sleep is an active 
process rather than a passive process such as a suppression of wakefulness. For example, orexin, an endogenous wakefulness-promoting substance, also facilitates the emergence from anesthesia, but not the delayed induction of anesthesia [2, 9-11]. Another endogenous wakefulness-promoting substance, neuropeptide $S$, also facilitates the emergence from anesthesia, but not induction [12]. These results may confirm a theory that explains the process of anesthesiainduced loss of consciousness and emergence as not being a simple mirror of the unitary mechanism and, moreover, that these processes are diverse depending on the anesthetic agent. For example, in rats as well as humans, propofol and dexmedetomidine produce a calm induction of anesthesia and emergence. Conversely, anesthesia and emergence are relatively unstable with ketamine. Indeed, rats maintain a state of wakefulness for several hours without any sleep-like behavior after emergence from ketamine anesthesia [13].

\section{Anesthesia and sleep: from the clinical aspect}

1. Sleep disorders after general anesthesia are harmful.

Anesthesia and surgical stress disturb sleep rhythms [14]. Although wound pain itself is an important causal factor [15], opioids also disturb sleep, especially REM sleep [16, 17]. This sleep disturbance can adversely affect the postoperative course. First, sleep disorders suppress the immune function, which increases the risk of infection and cancer progression. In addition, sleep disorders impair autonomic nervous function. REM sleep disorders result in the disorchestration of autonomic nervous activity, which can lead to a serious ischemic condition in the cerebrovascular or cardiovascular system, and respiratory dysfunction such as bronchial asthma. Moreover, sleep disorders impair higher brain functions, such as memory storage, and can cause cognitive dysfunction. Patients with postoperative cognitive dysfunction have a high mortality rate within 1 year.

Previously, these sleep disturbance-related complications have been reported to occur on the 3rd and 4th postoperative days; however, they have been reported to develop as early as the 1 st or 2 nd day in recent years. Several short-acting anesthetics are clinically available, and are able to shorten the recovery process. As a result, complications tend to develop during the early period. The shortening of the recovery process itself is welcome, but it is worth noting that we still need medical resources for the treatment of the complications. From the point of view of medical economy and safety, sleep disturbance is a problem that should not be overlooked. To facilitate a smooth recovery from anesthesia and surgery, the above-mentioned postoperative complications should be prevented, which would be of great benefit to the medical economy. It can also be expected that patients will have a favorable postoperative course. Therefore, the most important issue is to establish a method of anesthesia that causes as little sleep disorder as possible after surgery.

\section{Do general anesthetics replace sleep?}

This depends on the type of general anesthetic. Inhaled anesthetics (e.g., enflurane, sevoflurane, isoflurane, desflurane) do not appear to be an alternative to sleep [18]. Based on my own experience, despite my surgery being only a minor one in ophthalmology, I felt fatigued after emerging from enflurane anesthesia, and I could not recognize the time course during anesthesia. Inhaled anesthetics may not provide a deep sleep-like effect on the patient. On the other hand, propofol may prevent sleep disturbance [19]. In addition, dexmedetomidine may partially provide some supplemental sleep [20]. Thus, if we can provide patients with sleep disturbance-free anesthesia, we will also have an anesthesia which is free from severe postoperative complications. Disturbance-free sleep is a concept that is not limited to only the perioperative period but also can be applied to many pathological conditions including cases in the intensive care unit. In developing such a method of anesthesia, there are many issues that must be solved; nevertheless, this field is promising.

The following is a summary of the above:

1. In general, the term general anesthesia does not distinguish between pharmacologically different phenomena.

2. Sleep shares some common mechanisms with general anesthesia-induced loss of consciousness.

3. Studies on sleep may elucidate the mechanism concerning how general anesthesia induces loss of consciousness.

4. The process of anesthesia-induced loss of consciousness and emergence is not a simple mirror of the unitary mechanism.

5. Sleep disorders following general anesthesia are harmful.

a. Suppressed immunity results in infection and the exacerbation of cancer.

b. REM sleep disorder leads to an imbalance of autonomic nervous activity, resulting in a wide range of serious disorders in the respiratory and circulatory systems such as ischemic cerebrovascular disease, heart disease, and bronchial asthma.

c. Higher brain functions are also impaired. As a result, memory and cognitive impairment may occur. Patients who have cognitive impairment after surgery have a high mortality rate within 1 year. 
6. Do general anesthetics replace sleep?

a. Inhalation anesthetics cannot be an alternative.

b. GABA receptor agonists (propofol) and $\alpha 2$ adrenergic receptor agonists (dexmedetomidine) may partially serve to complement sleep.

Anesthesiology is a field that is interdisciplinary. As part of a cross-disciplinary approach, incorporating sleep science research into anesthesiology will contribute to developing the knowledge base of anesthesiology.

Funding This article was supported by funds from the Ministry of Education, Culture, Sports, Science and Technology of Japan (Grantsin-aid for scientific research: 26462327 and 18K08807).

\section{References}

1. Hameroff $\mathrm{S}$, Penrose R. Consciousness in the universe: a review of the 'Orch OR' theory. Phys Life Rev. 2014;11:39-78.

2. Kelz MB, Sun Y, Chen J, Cheng Meng Q, Moore JT, Veasey SC, Dixon S, Thornton M, Funato H, Yanagisawa M. An essential role for orexins in emergence from general anesthesia. Proc Natl Acad Sci U S A. 2008;105:1309-14.

3. Mashour GA, Kelz MB. Systems neuroscience: the exciting journey to oblivion. Curr Biol. 2018;28:R223-4.

4. Nelson LE, Guo TZ, Lu J, Saper CB, Franks NP, Maze M. The sedative component of anesthesia is mediated by GABA(A) receptors in an endogenous sleep pathway. Nat Neurosci. 2002;5:979-84.

5. Baker R, Gent TC, Yang Q, Parker S, Vyssotski AL, Wisden W, Brickley SG, Franks NP. Altered activity in the central medial thalamus precedes changes in the neocortex during transitions into both sleep and propofol anesthesia. J Neurosci. 2014;34:13326-35.

6. Song XX, Yu BW. Anesthetic effects of propofol in the healthy human brain: functional imaging evidence. J Anesth. 2015;29:279-88.

7. Uygun DS, Ye Z, Zecharia AY, Harding EC, Yu X, Yustos R, Vyssotski AL, Brickley SG, Franks NP, Wisden W. Bottom-up versus top-down induction of sleep by zolpidem acting on histaminergic and neocortex neurons. J Neurosci. 2016;36:11171-84.
8. Akeju O, Brown EN. Neural oscillations demonstrate that general anesthesia and sedative states are neurophysiologically distinct from sleep. Curr Opin Neurobiol. 2017;44:178-85.

9. Kushikata T, Hirota K, Yoshida H, Kudo M, Lambert DG, Smart D, Jerman JC, Matsuki A. Orexinergic neurons and barbiturate anesthesia. Neuroscience. 2003;121:855-63.

10. Shirasaka T, Yonaha T, Onizuka S, Tsuneyoshi I. Effects of orexin-A on propofol anesthesia in rats. J Anesth. 2011;25:65-71.

11. Zhang LN, Yang C, Ouyang PR, Zhang ZC, Ran MZ, Tong L, Dong HL, Liu Y. Orexin-A facilitates emergence of the rat from isoflurane anesthesia via mediation of the basal forebrain. Neuropeptides. 2016;58:7-14.

12. Kushikata T, Yoshida H, Kudo M, Salvadori S, Calo G, Hirota K. The effects of neuropeptide $\mathrm{S}$ on general anesthesia in rats. Anesth Analg. 2011;112:845-9.

13. Kushikata T, Sawada M, Niwa H, Kudo T, Kudo M, Tonosaki M, Hirota K. Ketamine and propofol have opposite effects on postanesthetic sleep architecture in rats: relevance to the endogenous sleep-wakefulness substances orexin and melanin-concentrating hormone. J Anesth. 2016;30:437-43.

14. Rosenberg-Adamsen S, Kehlet H, Dodds C, Rosenberg J. Postoperative sleep disturbances: mechanisms and clinical implications. Br J Anaesth. 1996;76:552-9.

15. Chouchou F, Khoury S, Chauny JM, Denis R, Lavigne GJ. Postoperative sleep disruptions: a potential catalyst of acute pain? Sleep Med Rev. 2014;18:273-82.

16. Cheatle MD, Webster LR. Opioid therapy and sleep disorders: risks and mitigation strategies. Pain Med. 2015;16(Suppl 1):S22-6.

17. Garcia AN, Salloum IM. Polysomnographic sleep disturbances in nicotine, caffeine, alcohol, cocaine, opioid, and cannabis use: a focused review. Am J Addict. 2015;24:590-8.

18. Nelson AB, Faraguna U, Tononi G, Cirelli C. Effects of anesthesia on the response to sleep deprivation. Sleep. 2010;33:1659-67.

19. Tung A, Bergmann BM, Herrera S, Cao D, Mendelson WB. Recovery from sleep deprivation occurs during propofol anesthesia. Anesthesiology. 2004;100:1419-26.

20. Su X, Wang DX. Improve postoperative sleep: what can we do? Curr Opin Anaesthesiol. 2018;31:83-8.

Publisher's Note Springer Nature remains neutral with regard to jurisdictional claims in published maps and institutional affiliations. 